\title{
Isquemia intestinal inducida por 5-fluorouracilo adyuvante en un paciente con adenocarcinoma de colon: reporte de caso y revisión de la literatura
}

\author{
- Mauricio Luján Piedrahíta', Sergio Mejía Espinosa²
}

${ }^{1}$ Clínica de oncología Astorga, Universidad Pontificia Bolivariana, Medellín.

2 Universidad Pontificia Bolivariana, Medellín.

\begin{abstract}
Paciente masculino de 59 años, posoperatorio de hemicolectomía derecha por adenocarcinoma de colon pT4aNOMO, quien inicia tratamiento con quimioterapia adyuvante, esquema 5 -fluorouracilo más ácido folínico (dosis de $425 \mathrm{mg} / \mathrm{m} 2$ y $20 \mathrm{mg} / \mathrm{m} 2$ intravenosos día 1 a 5, cada 28 días por 6 ciclos). En el día 3 del primer ciclo, el paciente ingresa a sala de quimioterapia refiriendo dolor abdominal severo y vómito; al examen físico con signos de irritación peritoneal, taquicardia e hipotensión; se envía a urgencias, donde es evaluado por servicio de cirugía, por lo que fue llevado a laparotomía exploratoria, requiriendo resección de $60 \mathrm{~cm}$ de íleon. El estudio anatomopatológico reveló necrosis isquémica de íleon. Se decidió dada la complicación suspender la quimioterapia y continuar seguimiento clínico.
\end{abstract}

\section{Características epidemiológicas e histopatológicas del adenocarcinoma colorrectal en Cartagena de Indias (norte de Bolívar, Colombia), 2009-2011}

\section{Eusebio Enrique Contreras Borrego, Haroldo Estrada López, Ismael Yépez Barreto}

Facultad de Medicina, Universidad de Cartagena.

Grupo de Investigación Prometeus.

\begin{abstract}
Objetivo. Describir las características epidemiológicas e histopatologías del adenocarcinoma colorrectal en Cartagena de Indias (Colombia) entre los años 2009 y 2011.

Metodología. Fueron revisados retrospectivamente los archivos y bases de datos de todos los servicios de anatomía patológica en Cartagena de Indias en búsqueda de todos los casos de adenocarcinoma colorrectal entre el $1^{\circ}$ de enero de 2009 y el 31 de enero de 2011. Se estimó la tasa cruda (TC) de incidencia anual por cada 100.000 habitantes, la tasa ajustada por edad se calculó por el método directo utilizando la población estándar propuesta por Doll et al. (IARC, 1966).

Resultados. Se encontraron 370 casos (112 en 2009, 124 en 2010 y 134 en 2011), de los cuales el 53,8\% son mujeres y el $46,2 \%$, hombres. En todos se determinó la edad, que osciló entre los 25 y 69 años, con un promedio de 63,3 (desviación estándar: 14,6). La tasa cruda (TC) de incidencia anual es de 25,93/100.000 habitantes y la tasa ajustada por edad (TAE) de 9,58/100.000 habitantes (IC95\% 25,81-31,91). La localización tumoral fue del $32,2 \%$ en recto y del $67,8 \%$ en colon. La diferenciación histológica fue: un 32,2\% buena, un 53,5\% moderada y un $14,3 \%$ pobre. La arquitectura mucinosa y las células en anillo de sello se reportaron en el $11,9 \%$ y $2,4 \%$, respectivamente. El 57,2\% de los casos reportó estudio en espécimen de resección quirúrgica, de estos, el 79,8\% compromete la serosa-grasa adyacente; el 56,6\%, los ganglios linfáticos; y el 59,8\%, la invasión linfovascular.

Conclusión. En Cartagena de Indias, la mayoría de casos reportados de adenocarcinoma colorrectal durante los años 2009-2011 corresponde a estadios avanzados de la enfermedad.
\end{abstract}

